\title{
Imaging of corrosion pits on metallic pipes using a laser scanner
}

\author{
Homayoun Najjaran
}

National Research Council, Ottawa, Ontario, Canada

This paper describes the hardware and software of a laser scanning system that is used to produce 3D images of external surfaces of pipes. The images are produced in the form of $3 \mathrm{D}$ raster images with a resolution of up to $0.1 \times 0.1 \mathrm{~mm}$ and an accuracy of 3-10 microns, depending on the desired scanning speed, to portray the corrosion pits on the pipes. The main application of the scanner is to establish patterns for calibrating nondestructive testing techniques (e.g., Remote Field Eddy Current (RFEC) and ultrasound testing that are commonly used to measure the remaining wall thickness of ductile and cast iron pipes), and also identifying the statistical characteristics of the measuring instruments utilized in those methods. The images may also be useful to scrutinize corrosion and failure mechanisms, especially when estimates of average or maximum pit depth are insufficient.

The scanning system consists of a 2-DOF robot that can move a laser displacement sensor along a pipe and an instrumented rig that rotates the pipe about its axis. Rotating the pipe and moving the rangefinder along the pipe's axis, the scanning system acquires laser measurements into a host computer to produce the image. The paper also presents the images and statistical analysis of corrosion pits of pipe samples exhumed and sandblasted for scanning. 